\title{
Propiedades Fisicoquímicas y de Flujo de un Yogur Asentado Enriquecido con Microcápsulas que Contienen Ácidos Grasos Omega 3
}

\author{
Reyes C. Macedo y Ramírez y Jorge F. Vélez-Ruíz \\ Departamento de Ingeniería Química, Alimentos y Ambiental. Universidad de Las Américas Puebla., \\ Ex hacienda Santa Catarina Mártir, San Andrés Cholula, Puebla. C.P. 72810, México \\ (e-mail: reyes.macedorz@udlap.mx; jorgef.velez@udlap.mx)
}

Recibido Mar. 19, 2015; Aceptado May. 8, 2015; Versión final Jun. 19, 2015, Publicado Oct. 2015

\begin{abstract}
Resumen
Se han estudiado y analizado las propiedades fisicoquímicas y de flujo de un yogur enriquecido con ácidos grasos poli-insaturados omega-3 en microcápsulas a dos concentraciones y almacenado durante 3 semanas en refrigeración. Los sistemas preparados fueron analizados semanalmente. Con respecto al factor de concentración, la sinéresis disminuyó y los valores de grados Brix aumentaron. Con respecto al tiempo de almacenamiento, los valores de $\mathrm{pH}$ disminuyeron, la humedad y la densidad permanecieron estables, el color mostró tendencia hacia el amarillo, perdiendo escasa luminosidad. Las propiedades de flujo mostraron un comportamiento no-Newtoniano de tipo seudoplástico. Los datos fueron ajustados a los modelos de Herschel-Bulkley y Ley de Potencia, permitiendo establecer la correspondiente relación experimental de los tres parámetros de flujo con las variables de estudio. El yogur favorece su funcionalidad y ciertas características fisicoquímicas con la adición de omega-3, además de su consistencia, viscosidad y color.
\end{abstract}

Palabras clave: yogur, ácidos grasos, microcápsulas, propiedades fisicoquímicas, ley de potencia

\section{Physicochemical and Flow Properties of a Seated Yogurt Enriched with Microcapsules containing Omega 3 Fatty Acids}

\begin{abstract}
Physicochemical and flow properties of a yogurt enriched with polyunsaturated fatty acids omega- 3 in microcapsules, at two concentrations and during 3 weeks of cold storage, were studied and analyzed. The prepared systems were analyzed every week. Syneresis phenomenon decreased while ${ }^{\circ}$ Brix increased as a function of microcapsules concentration. With respect to storage, $\mathrm{pH}$ values decreased, moisture and density were stable, color showed tendency to yellow with low loss of lightness. Flow properties showed a non-Newtonian behavior of pseudoplastic type. Data were fitted to the Herschel-Bulkley and Power Law models that allow establishing the corresponding relation of the three experimental flow parameters with respect to the studied variables. The yogurt favors its functionality by the addition of omega- 3 and improves some of its physicochemical characteristics, besides its consistency, viscosity and color.
\end{abstract}

Keywords: yogurt, fatty acids, microcapsules, physicochemical properties, power law 


\section{INTRODUCCIÓN}

Generalmente el yogur es un producto elaborado con leche tratada térmicamente y puede también ser homogeneizada antes de la inoculación de las bacterias ácido lácticas, Lactobacillus bulgaricus y Streptococcus thermophilus, (US FDA-DHHS, 2011). El yogur, que es un producto ampliamente consumido se presenta en el mercado en dos formas, asentado y batido; en ellos y principalmente en el asentado, tanto la consistencia como la sinéresis son dos propiedades de gran importancia para el consumidor y para el productor (Tamime y Robinson, 1999; Vélez-Ruiz y Rivas, 2001; Ramirez-Santiago et al., 2010; Vélez-Ruiz et al., 2012). Las propiedades fisicoquímicas, la textura y las propiedades de flujo están influenciados por varios factores, tales como la composición y formulación de la leche, el tratamiento térmico dado a la leche, la combinación de las bacterias ácido lácticas usadas, la velocidad de acidificación de la leche y el tiempo de almacenamiento (Dello et al., 2004; Sodine et al., 2004; Purwandari et al., 2007; Vélez-Ruiz, 2008; Özer y Kirmaci, 2010; Vélez-Ruiz et al., 2012; Tamjidi et al., 2012; Ramírez-Sucre y Vélez-Ruíz, 2013). El yogur además de ser un excelente alimento de origen lácteo, ha sido utilizado como un medio adecuado para la incorporación de otros nutrientes como componentes alimenticios, ingredientes prebióticos, fibra y calcio, entre otros (Aportela-Palacios et al., 2005; Vélez-Ruiz, 2008; Tamjidi et al., 2012; Vélez-Ruiz et al., 2012).

La incorporación de nuevos ingredientes en la composición de un yogur contribuye a modificar las propiedades y características de este producto lácteo (Vélez-Ruiz y Rivas, 2001; Vélez-Ruiz, 2008). Actualmente se ha incrementado la demanda por componentes bioactivos naturales, que preservan la salud y reducen los riesgos de enfermedades. Los efectos benéficos de los ácidos grasos poli-insaturados como el ácido eicosapentanoico (EPA; C20:5; n-3) y el ácido docosahexanoico (DHA; C22:6; n-3) están bien documentados, mostrando varios beneficios en la salud humana, incluyendo una reducción en los riesgos de enfermedades cardiovasculares, actividad anticancerígena, efectos antiinflamatorios, prevención de osteoporosis y disturbios neurológicos, también ayudan a reducir la depresión (Abeywardena y Head, 2001; McLennan y Abeywardena, 2005; Riediger et al., 2009; Wendel y Heller, 2009; Weitz et al., 2010; Tamjidi et al., 2012;).

Los ácidos grasos poli-insaturados omega-3 son altamente susceptibles a la oxidación y para consumirlo en la ingesta diaria, es necesario aplicar técnicas novedosas de protección como la microencapsulación, que mantienen su estabilidad y viabilidad en el tiempo. Este factor asociado a la resistencia de varios grupos de consumidores, a comer alimentos que son fuente de omega-3, como es el pescado, ha propiciado la aplicación de la microencapsulación en la industria de alimentos por medio del secado en espreas como una técnica muy eficiente, que facilita la incorporación de éstos y otros ingredientes en la formulación de nuevos productos alimenticios (Ackman, 2006; Saenz et al., 2009; Sathivel y Kramer, 2010; González et al., 2014). Y aunque normalmente, el enriquecimiento con componentes bioactivos no suministra los niveles nutricionales requeridos, si contribuye a un mejor estado de salud del ser humano. La sociedad internacional para el estudio de ácidos grasos y lípidos (ISSFAL por sus siglas en Inglés) recomienda una ingesta de EPA y DHA de al menos $500 \mathrm{mg} / \mathrm{d}$ (Cunnane et al., 2004), aunque en algunos países se recomiendan ingestas menores (300 a $500 \mathrm{mg} / \mathrm{d}$ ). Por lo que el objetivo del presente trabajo fue desarrollar un yogur que contenga ácidos grasos omega-3, EPA y DHA en microencapsulación, así como evaluar sus propiedades fisicoquímicas y de flujo como función de dos variables de estudio, la concentración de ácidos grasos insaturados y el tiempo de almacenamiento.

\section{MATERIALES Y MÉTODOS}

\section{El aceite de pescado.}

El aceite de hígado de pescado de bacalao blanco fue suministrado por Central de Drogas. SA de CV, Naucalpan Estado de México. Se protegió de la luz y se almacenó a temperatura de $4^{\circ} \mathrm{C}$. El valor de peróxido fue determinado siguiendo el método Cd 8-53 del ácido acético:cloroformo (AOCS, 1997), el cual se reporta en mili equivalentes de peróxido por $1000 \mathrm{~g}$ de aceite.

\section{Microcápsulas con aceite de hígado de pescado de bacalao blanco, fuente de omega-3}

Estas fueron obtenidas siguiendo el proceso descrito por Sathivel y Kramer (2010) y Estrada et al. (2011). Una emulsión estable fue preparada utilizando un procesador ultrasónico CPX-500 (Cole Palmer Instruments, Vernon Hills, EU) y empleando la siguiente relación de componentes: $7 \%$ de aceite de pescado, $22 \%$ de goma arábiga, $11 \%$ de maltodextrina y $60 \%$ de agua. La emulsión fue encapsulada en un secador por aspersión B290 (Buchi Mini Spray Dryer, Labortechnick AG, Suiza) a una temperatura del aire de entrada de $180^{\circ} \mathrm{C}$. Se determinó la cantidad de aceite de pescado en las microcápsulas, aplicando el método Aa 4-38 a una muestra de $2 \mathrm{~g}$ de microcápsulas, y se reporta como porcentaje ( $\mathrm{p} / \mathrm{p})$ de aceite en la muestra (AOCS, 1997).

Preparación del yogur con microcápsulas 
El yogur natural se elaboró siguiendo el método de Tamime y Robinson (1999). Leche entera liquida se estandarizó a $16 \%(\mathrm{p} / \mathrm{p})$ de sólidos con leche entera en polvo, se pasteurizó a $90^{\circ} \mathrm{C}$ durante 10 min, posteriormente se enfrió hasta $42^{\circ} \mathrm{C}$, para realizar la adición de los microorganismos liofilizados (Danisco S.A., $\mathrm{DF}$, México) en forma directa en una relación de $3 \mathrm{mg} / \mathrm{L}$ de leche, la incubación se realizó durante $6 \pm 1 \mathrm{~h}$ hasta alcanzar un $\mathrm{pH}$ de $4.5 \pm 0.1$, para su posterior enfriamiento a $5 \pm 1^{\circ} \mathrm{C}$. Adicionándolo posteriormente con $2.7 \%$ y $5.4 \%$ de microcápsulas y considerando que solo es una incorporación parcial, para no saturar al yogur, las muestras fueron enfriadas inmediatamente y almacenadas a $4^{\circ} \mathrm{C}$ en un refrigerador.

La manera de identificar los yogures es la siguiente: AM (yogur asentado, adicionado con microcápsulas), los números 1, 8, 15, 22 representan los días de almacenamiento, y los números siguientes 0,27 y 54 representan las concentraciones de $0,2.7$ y 5.4\% de microcápsulas adicionadas respectivamente. Así la muestra AM10 corresponde al sistema del primer día con cero \% de microcápsulas; la muestra AM827 indica una muestra de yogur del día 8 con $2.7 \%$ de microcápsulas adicionadas, mientras que el sistema AM2254 indica una muestra de yogur del día 22 con $5.4 \%$ de microcápsulas adicionadas, y así correspondientemente. Los sistemas fueron almacenados por 3 semanas y analizados por triplicado en ${ }^{\circ} \mathrm{Bx}, \mathrm{pH}$, acidez, sinéresis, humedad, densidad, color y propiedades de flujo, en los días 1, 8, 15 y 22. Para conocer las concentraciones de ácidos grasos insaturados en el aceite de pescado, se utilizó la información proporcionada por el proveedor, 20\% mínimo de acidos grasos poliinsaturados (PUFA), 0.9 es material insaponificable y el resto son ácidos grasos (Central de Drogas, S.A.); así como el balance de materia y análisis de grasa aplicados en los yogures preparados para este estudio (en $\mathrm{mg} / 100 \mathrm{~g}$ ), por lo que se presenta la tabla 1.

Tabla 1. Contenido de ácidos grasos insaturados en el aceite de pescado de bacalao blanco y de los sistemas de yogur estudiados. * Central de Drogas, S.A., para $100 \mathrm{~g}$ de aceite. ${ }^{* *}$ Para $100 \mathrm{~g}$ de yogur.

\begin{tabular}{cccccc}
\hline Componente & Aceite (\%)* & $\begin{array}{c}\text { Microcápsulas } \\
\text { en yogur (\%) }\end{array}$ & $\begin{array}{c}\text { EPA } \\
\mathrm{mg}\end{array}$ & $\begin{array}{c}\mathrm{DHA} \\
\mathrm{mg}\end{array}$ & $\begin{array}{c}\mathrm{EPA}^{\mathrm{D}} \mathrm{DHA} \\
\mathrm{mg}\end{array}$ \\
\hline Ácidos grasos libres & 0.06 & - & - & - & - \\
Ácido Eicosapentaenoico (EPA) & 9.60 & 2.7 & 4.81 & 6.21 & 11.02 \\
Ácido Docosahexaenoico (DHA) & 11.30 & 5.4 & 9.61 & 12.42 & 22.03 \\
\end{tabular}

\section{Análisis fisicoquímicos}

Los sólidos solubles expresados como ${ }^{\circ} \mathrm{Bx}$, se determinaron con un refractómetro digital (Reitcher AR 200, Waltham MA, EU), usando el método 932.12 (AOAC, 2000). El pH fue medido usando un potenciómetro digital Corning modelo 445 (Corning Incorporate, NY, EU).), el instrumento fue calibrado usando soluciones estándar con $\mathrm{pH}$ de 4.0 y 7.0. La acidez se determinó por titulación potenciométrica, usando $\mathrm{NaOH} 0.1 \mathrm{~N}$ hasta pH 8.2 y se expresó como porcentaje $(\mathrm{g} / 100 \mathrm{~g})$ de ácido láctico, de acuerdo al método 942.15 (AOAC, 2000). La capacidad de retención de agua del yogur expresada como sinéresis, fue determinada aplicando la técnica de Kessler (1998), usando una centrífuga Adams (Clay Adams Inc., Chatsworth, CA, EU) a 5000 rpm durante $20 \mathrm{~min}$. Con este procedimiento el suero es separado y pesado, la sinéresis se expresa en porcentaje.

$$
\% \text { sinéresis }=\left(m_{\text {suero }}\right)(100) / m_{\text {muestra }}
$$

En esta ecuación $\mathrm{m}$ representa la masa del suero ( $\left.m_{\text {suero }}\right)$ en $\mathrm{g}$ y la masa de la muestra en $g$ ( $\left.m_{\text {muestra}}\right)$, respectivamente

Para la determinación de la humedad se utilizó el método 934.01 (AOAC, 2000), evaporando el agua de la muestra, se calculó por diferencia de pesos y se reporta en porcentaje (g/100 g de muestra). La densidad se determinó con picnómetros de aluminio de boca ancha, Gardner 07019 (Paul N Gardner, CA, EU). El color se determinó usando el Colorímetro Chroma meter CR 400 (Konica Minolta, Japón). Previamente calibrado con mosaicos negro y blanco ( $L$, a, b para el mosaico blanco de 92.89, -1.05 y 0.82), en modo de reflectancia. El cambio de color neto $(\Delta \mathrm{E})$, se determinó con la siguiente relación (Popov-Raljic et al., 2008):

$$
\Delta \mathrm{E}=\left[\left(\mathrm{L}-\mathrm{L}_{0}\right)^{2}+\left(\mathrm{a}-\mathrm{a}_{0}\right)^{2}+\left(\mathrm{b}-\mathrm{b}_{0}\right)^{2}\right]^{0.5}
$$

En esta ecuación $L$, $a$ y $b$ son los parámetros de Hunter medidos para el yogur o sistema a un cierto tiempo, $L_{0}, a_{0}$ y $b_{0}$ son los parámetros correspondientes para el yogur control.

Propiedades de flujo 
Las propiedades de flujo se determinaron a $20 \pm 1^{\circ} \mathrm{C}$, usando un viscosímetro digital Brookfield DV-III (Brookfield Engineering Laboratories Inc., Middlebore, MA, EU), que es un instrumento rotacional, utilizando dos agujas de prueba y el adaptador de muestra pequeña $(10-11 \mathrm{~mL})$. El esfuerzo cortante $(\tau)$ fue determinado a la correspondiente razón de corte o deformación $(\gamma)$ con diferentes velocidades de giro aplicando una rampa de $120 \mathrm{~s}$. Las mediciones fueron realizadas los días 1, 8, 15 y 22. Los datos experimentales se ajustaron al modelo de Ley de Potencia (LP, ecuación 3) y al modelo de Herschel y Bulkley (HB, ecuación 4).

$$
(\tau=K y \cdot n)
$$

En esta ecuación $\tau$ representa el esfuerzo cortante en $\mathrm{Pa}, K$ representa el coeficiente de consistencia en $\mathrm{Pa} \cdot \mathrm{s}^{n}, \dot{\gamma}$ representa la velocidad de corte en $1 / \mathrm{s}$ y $n$ representa el índice de comportamiento al flujo, adimensional.

$$
\left(\tau=\tau_{0}+K \dot{r} \dot{n}\right)
$$

En esta ecuación $\tau_{0}$ representa el esfuerzo de cedencia o umbral de fluencia en $\mathrm{Pa}, \tau, \gamma, K$ y $n$ tienen el mismo significado que en la ecuación 3.

Los tres parámetros, esfuerzo de cedencia o umbral de fluencia $\left(\tau_{0}\right)$, índice de flujo $(n)$ y coeficiente de consistencia $(K)$ de estos modelos reológicos, y también el valor de la viscosidad aparente (ecuación 5), fueron utilizados para caracterizar el comportamiento al flujo de las muestras de yogur. El esfuerzo de cedencia se calculó usando el modelo de Casson (ecuación 6). Posteriormente, se realizó una correlación lineal del log de $\left(\tau\right.$ - $\left.\tau_{0}\right)$ vs log $\dot{\gamma}$, para determinar los parámetros de flujo (Ramírez-Sucre y Vélez-Ruíz, 2011)

$$
\begin{aligned}
& (\eta=\tau / \dot{Y}) \\
& \left(\tau^{1 / 2}=\tau_{0} 1 / 2+C \dot{\gamma}^{1 / 2}\right)
\end{aligned}
$$

En esta ecuación $\eta$ representa la viscosidad aparente en Pas, $C$ es una constante del modelo, $\tau$, $\tau$ o y $\dot{\gamma}$ representan los mismos parámetros que en la ecuaciones 3 y 4 .

Las ecuaciones empleadas en las pruebas de bondad de ajuste, la raíz cuadrada del error medio (RMSE por sus siglas en inglés, ecuación 7) y porcentaje de error medio (PEM por sus siglas en inglés, ecuación 8 ) fueron usadas para definir el modelo de flujo que mejor ajusta los datos experimentales.

$$
\begin{aligned}
& \text { RMSE }=\left[\frac{1}{n} \sum_{i=1}^{n}\left(\tau_{\exp }-\tau_{\text {pred }}\right)^{2}\right]^{1 / 2} \\
& P E M=\frac{100}{n} \sum_{i=1}^{n} \frac{\tau_{\text {exp }}-\tau_{\text {pred }}}{\tau_{\exp }}
\end{aligned}
$$

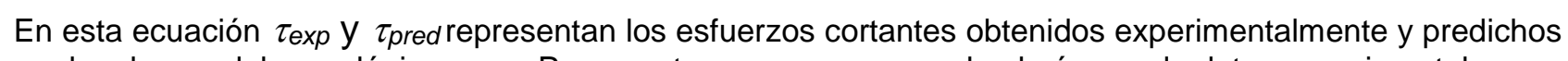
por los dos modelos reológicos en $\mathrm{mPa}$, en este caso $n$ corresponde al número de datos experimentales.

\section{Análisis estadístico}

El análisis de varianza y la regresión de superficie de respuesta, se realizó usando el paquete MINITAB (versión 16, Minitab Inc., State College, PA, EU), para determinar los efectos de la concentración de microcápsulas y el tiempo de almacenamiento sobre las muestras del yogur en sus propiedades fisicoquímicas y reológicas. El nivel de significancia fue de 5\%, aplicando la prueba de Tukey para diferenciar los sistemas.

\section{RESULTADOS Y DISCUSIÓN}

\section{Encapsulamiento del aceite de hígado de pescado de bacalao blanco}

El valor de peróxido (VP) como un indicador de la oxidación lipídica inicial, tiene un valor máximo recomendado para consumo humano de VP $\leq 10$ meq de peróxido/1000 g de muestra. El VP cuantificado en 
el aceite y en las microcápsulas fue 4.65 y 3.95 meq de peróxido/1000 g de muestra, respectivamente. De acuerdo a Gracey et al. (1999), aquellos aceites con un VP < $5 \mathrm{meq} / 1000 \mathrm{~g}$ de muestra se consideran frescos, por lo que ambos, aceite y cápsulas, resultaron aceptables para consumo humano.

\section{Determinación de ácidos grasos en microcápsulas.}

Usando $2 \mathrm{~g}$ de microcápsulas, como muestra y éter de petróleo como solvente, se determinó un promedio de $17.17 \%$ de aceite. Por otro lado, realizando un balance de materia en base seca, entre el flujo de alimentación y del producto en el secador empleado en la preparación de las cápsulas, se calculó un 17.48\% de aceite. Con estos datos como base y la composición del aceite de pescado, se obtuvo que $1.0 \mathrm{~g}$ de microcápsulas contiene alrededor de $35 \mathrm{mg}$ de EPA y DHA. Por lo que las muestras de yogur elaboradas con $5.4 \%$ y $2.7 \%$ de microcápsulas les corresponde alrededor de 200 y 100 mg de ácidos grasos insaturados respectivamente, en una porción de yogur de $100 \mathrm{~g}$; cumpliendo solo y respectivamente, con el 40 y $20 \%$ de los 500 mg/día recomendados por la ISSFAL; por lo que para satisfacer este requerimiento de ácidos grasos insaturados en la ingesta diaria, será necesario recurrir a otras fuentes de omega 3.

\section{Caracterización fisicoquímica}

Los resultados de la caracterización fisicoquímica de los yogures preparados y su evolución durante el almacenamiento se presentan en la Tabla 2. Las propiedades presentaron algunos cambios entre el primer día y el último día, el pH para el yogur control estuvo entre 4.88 y 4.53 , mientras que para el yogur con $5.4 \%$ de microcápsulas varió de 4.62 a 4.46, La presencia de las microcápsulas y el tiempo de almacenamiento tuvieron influencia significativa $(\mathrm{P}<0.05)$ en el $\mathrm{pH}$, lo que se atribuye además de la composición, a la acción de las bacterias ácido lácticas. Lebos et al. (2010) reportan un pH de 4.44 a 4.08 para yogur asentado, enriquecido también con probióticos microencapsulados, durante 21 días de almacenamiento. Otros autores como Estrada et al. (2011) reportan un pH de 4.5 a 4.4 para un yogur asentado fortificado con aceite de pescado microencapsulado durante cuatro semanas de almacenamiento. La acidez que está relacionada inversamente al pH, aumentó durante el almacenamiento desde 0.51 a $0.74 \mathrm{~g} / \mathrm{L}$ para los yogures control y de 0.52 a $0.69 \mathrm{~g} / \mathrm{L}$ para los yogures con $5.4 \%$ de microcápsulas. Los cambios de acidez son el resultado de las transformaciones bioquímicas desarrolladas en el yogur durante su almacenamiento, por lo que resultó que el tiempo si afectó significativamente $(\mathrm{P}<0.05)$ la acidez, pero no es el caso de la formulación. Tridjoko et al. (1992) reportan 0.7 - $1.35 \mathrm{~g} / \mathrm{L}$ de acidez para yogur con soya. Pirkul et al. (1998) reportan un rango de 0.85 $1.10 \mathrm{~g} / \mathrm{L}$ en yogur enriquecido con calcio.

Tabla 2: Propiedades fisicoquímicas de los yogures control y con microcápsulas. Valores son medias de 3 réplicas. Valores con letras superíndice a-g en la misma columna son significativamente diferentes $(p<0.05)$.

\begin{tabular}{ccccccc}
\hline Muestra & ${ }^{\circ} \mathrm{Bx}$ & $\mathrm{pH}$ & Acidez $(\mathrm{g} / \mathrm{L})$ & Sinéresis $(\%)$ & Humedad $(\%)$ & Densidad $\left(\mathrm{kg} / \mathrm{m}^{3}\right)$ \\
\hline AM10 & $12.40 \pm 0.10^{\mathrm{e}}$ & $4.88 \pm 0.01^{\mathrm{a}}$ & $0.51 \pm 0.01^{\mathrm{h}}$ & $41.90 \pm 0.70^{\mathrm{a}}$ & $87.42 \pm 0.92^{\mathrm{a}}$ & $1245.80 \pm 0.04^{\mathrm{a}}$ \\
AM127 & $16.46 \pm 0.06^{\mathrm{bc}}$ & $4.60 \pm 0.03^{\mathrm{bc}}$ & $0.51 \pm 0.00^{\mathrm{h}}$ & $36.24 \pm 0.48^{\mathrm{c}}$ & $81.51 \pm 1.86^{\mathrm{c}}$ & $1238.10 \pm 0.01^{\mathrm{a}}$ \\
AM154 & $16.46 \pm 0.15^{\mathrm{bc}}$ & $4.62 \pm 0.01^{\mathrm{b}}$ & $0.52 \pm 0.00^{\mathrm{g}}$ & $25.54 \pm 2.78^{\mathrm{d}}$ & $82.52 \pm 1.74^{\mathrm{bc}}$ & $1240.80 \pm 0.00^{\mathrm{a}}$ \\
AM80 & $12.13 \pm 0.40^{\mathrm{e}}$ & $4.40 \pm 0.01^{\mathrm{g}}$ & $0.57 \pm 0.00^{\mathrm{f}}$ & $40.88 \pm 1.27^{\mathrm{ab}}$ & $86.20 \pm 0.38^{\mathrm{ab}}$ & $1219.90 \pm 0.02^{\mathrm{a}}$ \\
AM827 & $16.00 \pm 1.65^{\mathrm{c}}$ & $4.48 \pm 0.02^{\mathrm{f}}$ & $0.56 \pm 0.01^{\mathrm{fg}}$ & $27.47 \pm 0.99^{\mathrm{d}}$ & $81.06 \pm 1.01^{\mathrm{c}}$ & $1233.90 \pm 0.00^{\mathrm{a}}$ \\
AM854 & $18.20 \pm 0.50^{\mathrm{a}}$ & $4.46 \pm 0.01^{\mathrm{f}}$ & $0.55 \pm 0.00^{\mathrm{g}}$ & $23.10 \pm 2.36^{\mathrm{ef}}$ & $82.34 \pm 1.02^{\mathrm{bc}}$ & $1241.70 \pm 0.01^{\mathrm{a}}$ \\
AM150 & $12.30 \pm 0.40^{\mathrm{e}}$ & $4.56 \pm 0.01^{\mathrm{de}}$ & $0.67 \pm 0.01^{\mathrm{d}}$ & $36.58 \pm 1.08^{\mathrm{c}}$ & $85.43 \pm 1.22^{\mathrm{abc}}$ & $1241.20 \pm 0.01^{\mathrm{a}}$ \\
AM1527 & $14.16 \pm 0.15^{\mathrm{d}}$ & $4.60 \pm 0.01^{\mathrm{bc}}$ & $0.64 \pm 0.01^{\mathrm{e}}$ & $29.21 \pm 1.67^{\mathrm{d}}$ & $84.62 \pm 1.26^{\mathrm{abc}}$ & $1233.20 \pm 0.00^{\mathrm{a}}$ \\
AM1554 & $16.30 \pm 0.00^{\mathrm{c}}$ & $4.60 \pm 0.00^{\mathrm{b}}$ & $0.63 \pm 0.01^{\mathrm{e}}$ & $21.17 \pm 0.12^{\mathrm{f}}$ & $84.44 \pm 1.15^{\mathrm{a}}$ & $1243.20 \pm 0.00^{\mathrm{a}}$ \\
AM220 & $12.23 \pm 0.15^{\mathrm{e}}$ & $4.53 \pm 0.01^{\mathrm{e}}$ & $0.74 \pm 0.01^{\mathrm{a}}$ & $42.45 \pm 0.12^{\mathrm{a}}$ & $86.05 \pm 1.01^{\mathrm{ab}}$ & $1238.70 \pm 0.01^{\mathrm{a}}$ \\
AM2227 & $13.56 \pm 0.31^{\mathrm{de}}$ & $4.57 \pm 0.00^{\mathrm{cde}}$ & $0.72 \pm 0.01^{\mathrm{b}}$ & $37.72 \pm 0.43^{\mathrm{bc}}$ & $85.34 \pm 3.22^{\mathrm{abc}}$ & $1243.70 \pm 0.02^{\mathrm{a}}$ \\
AM2254 & $18.00 \pm 0.62^{\mathrm{ab}}$ & $4.59 \pm 0.01^{\mathrm{bcd}}$ & $0.69 \pm 0.01^{\mathrm{c}}$ & $22.78 \pm 1.17^{\mathrm{ef}}$ & $81.11 \pm 1.66^{\mathrm{c}}$ & $1245.60 \pm 0.00^{\mathrm{a}}$ \\
\hline
\end{tabular}

Los valores de sinéresis obtenidos en el presente estudio variaron desde 21.17 hasta $42.45 \%$. En la misma tabla 2, se observa que el yogur control presenta mayor grado de sinéresis que los yogures con microcápsulas. Los componentes de las microcápsulas, particularmente la goma arábiga y la maltodextrina, favorecieron la acción de retención de agua, debido a que contribuyen al efecto de malla en la red tridimensional del gel formado en el yogur. La humedad de las muestras, fue mayor en el control (85.43 - 87.42\%) que en los yogures con microcápsulas (81.11-87.44\%), debido a la propia formulación. Obatolu et al. (2009) reportan una humedad de $83.8 \%$ para un yogur asentado normal. La densidad no presentó cambios significativos ( $P$ > 0.05) en las diferentes muestras, ni por efecto de las microcápsulas, ni por el tiempo de almacenamiento. 
Los parámetros obtenidos para el color se presentan en la Tabla 3. En donde se establece que los sistemas lucieron blancos y brillantes al ojo humano $(L>70)$, siendo más brillante el yogur control $(L>76)$. Aquellos sistemas que contienen microcápsulas mostraron un valor de luminosidad mayor a 70.72, mientras que $a$ y $b$ mostraron una tendencia hacia los colores verde y azul. El cambio neto del color $(\Delta \mathrm{E})$, resultó diferente en los yogures con microcápsulas y se incrementó con el almacenamiento, presentando diferencia significativa $(P<$ 0.05 ) entre el día 1 y 22, como consecuencia de los cambios bioquímicos del yogur. Pasephol et al. (2008) presentaron valores de 80.30 , - 2.2 y 1.3 para $L$, a y $b$ respectivamente, en yogur con inulina. Estrada et al. (2011) reportaron valores para un yogur sabor fresa, enriquecido con aceite de pescado después de cuatro semanas, con $82.5,11.4$ y 7.7 para los parámetros de color $L$, $a$ y $b$, respectivamente, que son comparables en $L$ y b a los yogures de este estudio.

Tabla 3: Cambios de color neto de los yogures durante el almacenamiento. Valores son medias de 3 réplicas. Valores con letras superíndice a-g en la misma columna son significativamente diferentes $(p<0.05)$.

\begin{tabular}{ccccc}
\hline Muestra $^{*}$ & $L$ & $a$ & $b$ & $\Delta \mathrm{E}$ \\
\hline AM10 & $77.89 \pm 1.07^{\mathrm{g}}$ & $-2.48 \pm 0.01^{\mathrm{f}}$ & $6.36 \pm 0.13^{\mathrm{g}}$ & 0.00 \\
AM127 & $71.32 \pm 0.30^{\mathrm{efg}}$ & $-2.24 \pm 0.03^{\mathrm{cd}}$ & $7.87 \pm 0.05^{\text {def }}$ & $2.91 \pm 0.28^{\text {def }}$ \\
AM154 & $70.72 \pm 0.33^{\mathrm{fg}}$ & $-2.28 \pm 0.03^{\mathrm{d}}$ & $7.69 \pm 0.04^{\mathrm{ef}}$ & $2.30 \pm 0.29^{\mathrm{ef}}$ \\
AM80 & $78.09 \pm 0.47^{\mathrm{abc}}$ & $-3.17 \pm 0.03^{\mathrm{d}}$ & $8.12 \pm 0.11^{\text {cde }}$ & $9.47 \pm 0.48^{\mathrm{abc}}$ \\
AM827 & $79.80 \pm 0.86^{\mathrm{a}}$ & $-2.74 \pm 0.02^{\mathrm{de}}$ & $8.88 \pm 0.05^{\mathrm{ab}}$ & $11.27 \pm 0.84^{\mathrm{a}}$ \\
AM854 & $79.48 \pm 0.18^{\mathrm{ab}}$ & $-2.56 \pm 0.04^{\mathrm{ef}}$ & $9.53 \pm 0.08^{\mathrm{a}}$ & $11.12 \pm 0.19^{\mathrm{ab}}$ \\
AM150 & $79.53 \pm 3.58^{\mathrm{ab}}$ & $-2.93 \pm 0.24^{\mathrm{a}}$ & $8.11 \pm 0.69^{\mathrm{cde}}$ & $10.88 \pm 3.65^{\mathrm{ab}}$ \\
AM1527 & $79.11 \pm 1.52^{\mathrm{abc}}$ & $-2.63 \pm 0.10^{\mathrm{ab}}$ & $8.38 \pm 0.26^{\mathrm{bcde}}$ & $10.50 \pm 1.48^{\mathrm{abc}}$ \\
AM1554 & $75.29 \pm 1.79^{\mathrm{bcde}}$ & $-2.31 \pm 0.07^{\mathrm{abc}}$ & $8.63 \pm 0.14^{\mathrm{bc}}$ & $6.86 \pm 1.74^{\mathrm{bcde}}$ \\
AM220 & $75.92 \pm 1.42^{\mathrm{abcd}}$ & $-2.62 \pm 0.05^{\mathrm{abc}}$ & $7.36 \pm 0.09^{f}$ & $7.19 \pm 1.42^{\mathrm{abc}}$ \\
AM2227 & $74.92 \pm 1.75^{\mathrm{cdef}}$ & $-2.53 \pm 0.17^{\mathrm{bcd}}$ & $8.04 \pm 0.31^{\text {cdef }}$ & $6.33 \pm 1.77^{\mathrm{cde}}$ \\
AM2254 & $71.64 \pm 0.13^{\text {defg }}$ & $-2.04 \pm 0.04^{\mathrm{cd}}$ & $8.45 \pm 0.06^{\mathrm{bcd}}$ & $3.50 \pm 0.12^{\text {def }}$ \\
\hline
\end{tabular}

\section{Comportamiento al flujo.}

Las curvas de flujo o reogramas obtenidas para todos los sistemas elaborados y almacenados, a la temperatura de $20 \pm 1^{\circ} \mathrm{C}$, se presentan en la Figura 1 donde se aprecia un comportamiento no-Newtoniano con diferente grado de plasticidad. En ella se aprecia en general, que el esfuerzo cortante y la viscosidad aumentaron con la concentración de aceite, como consecuencia de la fricción interna por la presencia de las microcápsulas o sus componentes; en contraste y como se esperaba, el yogur control registra los valores más bajos del esfuerzo y podría decirse que no muestra umbral de fluencia recién elaborado (primer día).

Las curvas muestran diferentes puntos experimentales debido a que hubo lecturas instrumentales en otro rango de corte, correspondientes a cada sistema, por ejemplo la muestra AM127 incluye 12 puntos, mientras que la muestra AM10 solo incluye 6 puntos. Cada sistema tuvo diferente respuesta y por ello el número de datos en la curva de flujo es diferente. Las viscosidades de los sistemas analizados mostraron valores de 1.97 mPa.s para el control (AM10), 6.19 y 7.83 mPa.s para los yogures enriquecidos con 2.7 (AM127) y 5.4\% (AM154) respectivamente, y fueron los valores correspondientes a una razón de corte de $11 \mathrm{~s}^{-1}$ para el día 1. $Y$ se observaron las siguientes tendencias en la viscosidad, aumenta al aumentar la concentración de microcápsulas, disminuye al aumentar la velocidad de deformación (pseudoplasticidad en un fluido no newtoniano) y disminuye con el tiempo de almacenamiento.

Las propiedades o parámetros de flujo obtenidos de las ecuaciones de Herschel-Bulkley y Ley de Potencia se incluyen en la Tabla 4. Considerando las pruebas de bondad de ajuste, se observa que los sistemas de yogur presentan un mejor ajuste a la Ley de Potencia. En la Tabla Los valores presentados son el promedio de tres réplicas. $\mathrm{K}$ es el coeficiente de consistencia $\left(\mathrm{mPa} \mathrm{s}{ }^{n}\right)$, $\mathrm{n}$ : índice de flujo (adimensional), $\tau_{0}$ : Esfuerzo de cedencia $(\mathrm{mPa})$. 


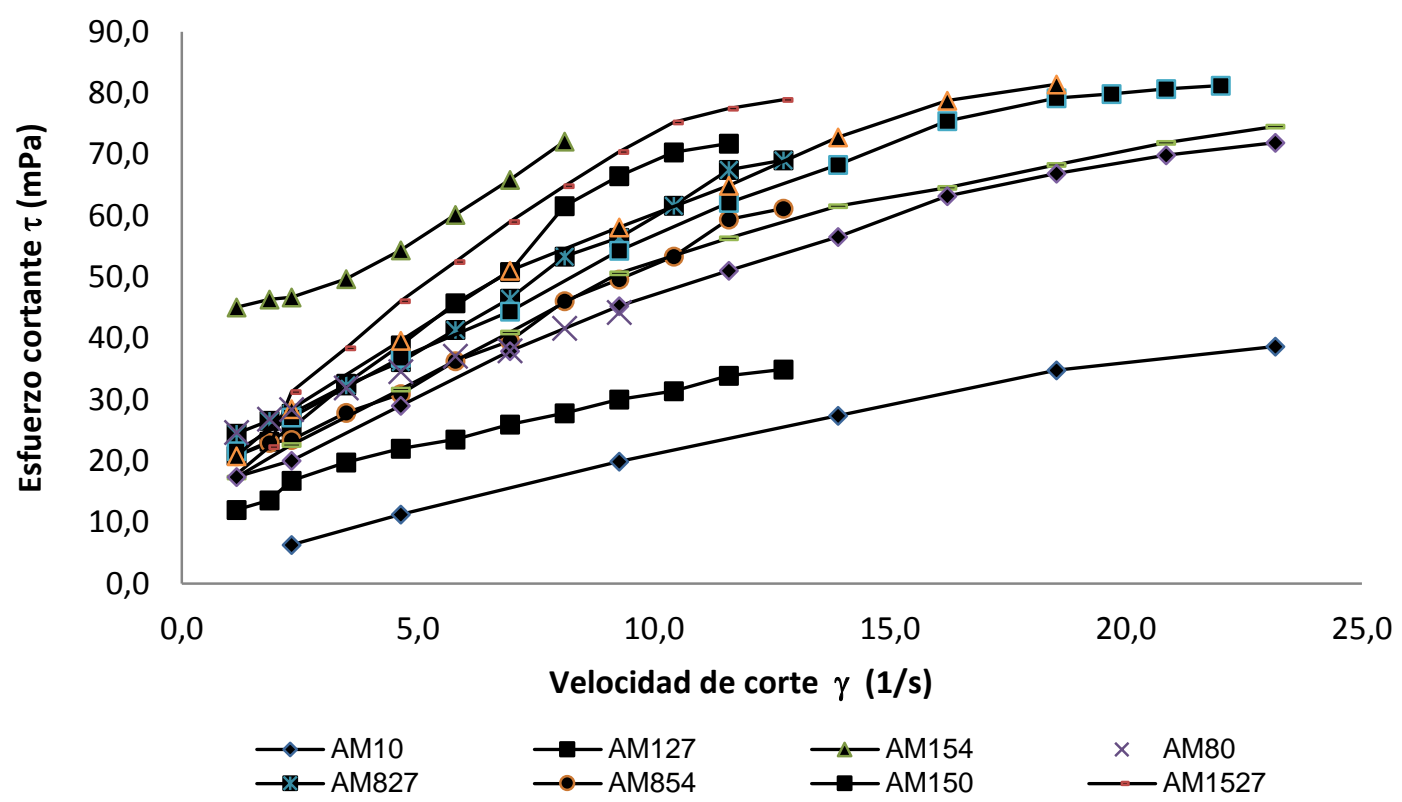

Fig. 1. Comportamiento al flujo de todos los sistemas de yogur, control y enriquecidos.

Tabla 4: Parámetros de flujo para los modelos de Herschel-Bulkley (HB) y Ley de Potencia (LP) de los diferentes sistemas de yogur estudiados, control y enriquecidos.

\begin{tabular}{ccccccccccccc}
\hline & \multicolumn{3}{c}{$\mathrm{HB}$} & \multicolumn{1}{c}{$\mathrm{LP}$} \\
\hline Muestra $^{*}$ & $\mathrm{~K}$ & $\mathrm{n}$ & -0 & $\mathrm{R}^{2}$ & $\mathrm{~K}$ & $\mathrm{n}$ & $\mathrm{R}^{2}$ & $\begin{array}{c}\text { RMSE } \\
\mathrm{HB}\end{array}$ & $\begin{array}{c}\text { PEM } \\
\mathrm{HB}\end{array}$ & $\begin{array}{c}\text { RMSE } \\
\text { LP }\end{array}$ & $\begin{array}{c}\text { PEM } \\
\text { LP }\end{array}$ \\
\hline & & & & & & & & & & & \\
AM10 & 0.17 & 0.84 & 1.70 & 0.99 & 3.55 & 0.76 & 1.00 & 9.43 & 7.23 & 0.34 & 0.03 \\
AM127 & 1.16 & 0.70 & 5.52 & 0.98 & 16.60 & 0.60 & 0.98 & 11.07 & 7.02 & 0.68 & -0.02 \\
AM154 & 1.22 & 0.53 & 28.80 & 0.92 & 39.61 & 0.25 & 0.89 & 8.51 & 4.80 & 0.95 & -0.02 \\
AM80 & 0.75 & 0.58 & 16.50 & 1.00 & 22.88 & 0.28 & 0.99 & 4.49 & 4.98 & 0.23 & -0.01 \\
AM827 & 1.12 & 0.63 & 9.74 & 0.97 & 19.71 & 0.47 & 0.96 & 8.19 & 4.50 & 0.77 & -0.09 \\
AM854 & 1.00 & 0.63 & 7.89 & 0.97 & 16.69 & 0.48 & 0.95 & 7.22 & 5.78 & 0.71 & -0.10 \\
AM150 & 0.53 & 0.67 & 6.34 & 0.99 & 11.02 & 0.45 & 0.99 & 4.68 & 5.32 & 0.13 & -0.01 \\
AM1527 & 1.12 & 0.77 & 6.10 & 0.98 & 16.71 & 0.64 & 0.99 & 12.17 & 6.43 & 0.54 & -0.01 \\
AM1554 & 0.77 & 0.72 & 9.26 & 0.99 & 15.40 & 0.51 & 0.99 & 11.28 & 5.79 & 0.44 & -0.01 \\
AM220 & 0.79 & 0.69 & 7.94 & 0.99 & 14.30 & 0.52 & 0.99 & 9.98 & 5.94 & 0.38 & -0.02 \\
AM2227 & 0.92 & 0.68 & 11.37 & 1.00 & 18.68 & 0.48 & 0.99 & 12.43 & 5.75 & 0.51 & -0.01 \\
AM2254 & 0.99 & 0.70 & 10.43 & 1.00 & 18.91 & 0.51 & 1.00 & 13.40 & 7.69 & 0.28 & -0.01 \\
\hline
\end{tabular}

La ventaja que tiene el modelo HB es que incorpora un parámetro de flujo más, el esfuerzo de cedencia; y a pesar de que no tiene el mejor ajuste, la diferencia de ajuste entre ambos modelos no es considerable. Existen diferentes estudios en yogur con adición de diferentes componentes alimenticos, en donde los ajustes son mejores en algunos casos a la LP, mientras que otros al modelo de HB (Dello et al., 2004; Aportela-Palacios et al., 2005; Paseephol et al., 2008; 2011; Vélez-Ruiz et al., 2012; Ramírez-Sucre y Vélez-Ruíz, 2013). El esfuerzo de cedencia como parámetro de flujo, representa la resistencia inicial al flujo ofrecida ya sea por formación de una estructura o por la acumulación de componentes sólidos, por lo que este parámetro se modifica en función de la concentración de sólidos y resulta mayor en el yogur enriquecido.

Tanto el coeficiente de consistencia como la viscosidad aparente de las muestras de yogur en todos los periodos de almacenamiento están influenciadas por los sólidos agregados que son de diferente tamaño y también por la desorganización de los agregados de las proteínas y los otros componentes, que aumentan con la velocidad de corte o deformación. Este comportamiento del fluido puede atribuirse a los efectos combinados de la ruptura de los enlaces débiles entre las proteínas de la leche y/o entre las proteínas de la 
leche y los ingredientes añadidos, microcápsulas con omega-3, así como la reintegración de tales uniones como resultado del movimiento Browniano y colisiones moleculares (Tang et al., 1993). Una disminución en la viscosidad aparente con un incremento en la velocidad de corte (pseudoplasticidad) también ha sido observada previamente en otros estudios, para yogur fortificado con salvado de trigo (Aportela-Palacios et al., 2005) y yogur con inulina (Paseephol et al., 2008), yogur con calcio y fibra (Vélez-Ruiz et al., 2012), así como yogur con cajeta (Ramírez-Sucre y Vélez-Ruíz, 2013), entre otros muchos estudios.Finalmente, para resumir el efecto de las variables de estudio y sus interacciones, tanto sobre las propiedades fisicoquímicas, como de flujo analizadas, se presenta la Tabla 5; la cual permite observar la significancia, analizada estadísticamente, de ambos factores (formulación: Form y tiempo de almacenamiento: Tpo), así como sus interacciones. Es interesante notar que el efecto es diverso.

Tabla 5: Efecto de los factores y sus interacciones por análisis estadístico en las propiedades reológicas del yogur con microcápsulas. $\mathrm{n}$ : índice de flujo (adimensional); K: coeficiente de consistencia $\left(\mathrm{Pa} \mathrm{S}^{\mathrm{n}}\right)$. ${ }^{*} \mathrm{~S}$ : significativo $(P<0.05)$, NS: no significativo $(P>0.05)$.

\begin{tabular}{|c|c|c|c|c|c|c|c|c|}
\hline $\begin{array}{l}\text { Factores e } \\
\text { Viscosidad } \\
\text { Interacciones* } \\
\text { aparente }\end{array}$ & Acidez & ${ }^{\circ} B x$ & Densidad & \multicolumn{3}{|c|}{ Humedad Sinéresis } & $n$ & $K$ \\
\hline Formulación & NS & $\mathrm{S}$ & NS & NS & $\mathrm{S}$ & NS & $S$ & $S$ \\
\hline Tiempo alm & $\mathrm{S}$ & NS & NS & $\mathrm{S}$ & $S$ & NS & NS & NS \\
\hline Form * Form & NS & NS & NS & NS & NS & NS & NS & $\mathrm{S}$ \\
\hline Tpo * Tpo & S & NS & NS & $\mathrm{S}$ & $\mathrm{S}$ & NS & NS & NS \\
\hline Form * Tpo & NS & NS & NS & NS & NS & $S$ & $S$ & $\mathrm{~S}$ \\
\hline
\end{tabular}

Con un nivel de confianza del $95 \%$ se puede asegurar que la formulación tuvo un efecto significativo $(\mathrm{P}<0.05)$ sobre la dulzura $0^{\circ} \mathrm{Bx}$, la sinéresis, el coeficiente de consistencia y la viscosidad aparente, del mismo modo se observa que no ejerció efecto significativo sobre el resto de las propiedades, destacando que no hubo efecto sobre el índice de flujo. Con respecto al factor tiempo de almacenamiento, se puede notar que no hubo efecto significativo ( $P>0.05$ ) sobre la mayoría de las propiedades (5 de 8); es de resaltar que no tuvo influencia sobre el coeficiente de consistencia, el índice de flujo y la viscosidad aparente. Acerca de las interacciones, la interacción $F^{*} F$ solo influyó en la viscosidad aparente; la interacción $T^{\star} T$ solo tuvo un efecto importante (significativo) en la acidez, la humedad y la sinéresis; mientras que la interacción de ambos factores $\left(\mathrm{F}^{\star} \mathrm{T}\right)$ afectó significativamente a las tres propiedades de flujo, coeficiente de consistencia, índice de flujo y viscosidad aparente. Y aunque se obtuvo un modelo por superficie de respuesta, éste no se incluyó.

En forma global se puede notar que las propiedades más afectadas correspondieron a las propiedades de flujo y la sinéresis. No se debe perder de vista, que las propiedades reológicas del yogur, en adición al efecto físico de la presencia de microcápsulas, también recibieron la influencia adicional de los hidrocoloides, por la solubilidad de la goma arábiga y la hidrólisis ácida de la maltodextrina presente en la membrana protectora de las microcápsulas (Valdés-Martínez, 2006).

\section{CONCLUSIONES}

El aceite de pescado con ácidos grasos insaturados omega-3, fue microencápsulado por medio de goma arábiga y maltodextrina, produciendo un polvo con partículas de tamaño uniforme y seco para ser adicionadas al yogur. Lo que facilitó su incorporación y distribución uniforme en la fase líquida y permitió la formulación de la bebida.

El presente trabajo, permitió desarrollar un yogur enriquecido con ácidos grasos poliinsaturados omega-3 como alimento potencial para el consumo y salud de los seres humanos. Contribuyendo con alrededor de un 20 a $40 \%$ de los requerimientos diarios de ácidos grasos, si se consume un yogur de $100 \mathrm{~g}$ con $2.7 \%$ y $5.4 \%$, respectivamente.

Las propiedades fisicoquímicas de los yogures elaborados presentaron valores propios de este producto y acordes a lo reportado en otros estudios. De estas propiedades, la sinéresis disminuyó en los sistemas enriquecidos, lo cual se considera como un valor agregado, ya que la presencia de las microcápsulas favoreció la retención de agua. Se atribuye a los hidrocoloides presentes en el envolvente de las microcápsulas, un efecto positivo importante, tanto en la sinéresis como en la consistencia del yogur. 
Tanto los sistemas de yogur enriquecido con omega-3, como el control, mostraron un comportamiento al flujo de tipo no-Newtoniano de naturaleza pseudoplástica o reoadelgazante, Aunque ambos modelos HB y LP, ajustaron bien el comportamiento al flujo del yogur, el ajuste a la LP fue mejor.

Las propiedades de flujo permitieron evaluar las características inherentes al yogur y observar los efectos de las variables de estudio en los tres parámetros: coeficiente de consistencia, índice de flujo y viscosidad, aparente, que son parámetros sumamente importantes en el manejo y en la calidad del producto.

La formulación tuvo efecto significativo en más de las propiedades analizadas (4 de 8), que el tiempo de almacenamiento (3 de 8); destacando la sinéresis y la consistencia afectadas por la formulación o presencia de microcápsulas con omega-3; así como la acidez y la sinéresis que fueron afectadas por el tiempo de almacenamiento.

\section{AGRADECIMIENTOS}

El autor Macedo-y-Ramírez agradece al Consejo Nacional de Ciencia y Tecnología (CONACYT) y a la Fundación Universidad de Las Américas Puebla (UDLAP) el apoyo financiero que me otorgan para la realización de los estudios de posgrado.

\section{REFERENCIAS}

Abeywardena, M.Y y Head, R.J., Longchain n-3 polyunsaturated fatty acids and blood vessel function, Cardiovascular Research: 52, 361-371 (2001)

Ackman, R.G., Marine lipids and omega-3 fatty acids, In C.C. Akoh, Handbook of Functional Lipids. CRC Press, Boca Raton, EU (2006)

AOAC. Official Methods of Analysis (17a Edición). Association of Official Analytical Chemist, Washington, EU (2000)

AOCS. Cd 8-53, Peroxide value acetic acid-chloroform method, Official Methods and Recommended Practices of the American Oil Chemists Society, Sampling and Analysis of Commercial Fats and Oils 1-2, EU (1997)

Aportela-Palacios, A., Sosa-Morales, M.E. y Vélez-Ruíz, J.F., Rheological and physicochemical behavior of fortified yogurt, with fiber and calcium. Journal of Texture Studies, 36, 333-349 (2005)

Cunnane S, Drevon C.A, Harris W, Sinclair A y Spector A. Recommendations for intakes of polyunsaturated fatty acids in healthy adults. ISSFAL Newsl. 11:12-25. (2004)

Dello, S.M., Bertola, N., Martino, M. y Bevilacqua, Y.A., Influence of dietary fiber addition on sensory and rheological properties of yoghurt, International Dairy Journal, 14, 263-268 (2004)

Estrada, J.D., Boeneke, C., Bechtel, P. y Sathivel, S., Developing a strawberry yogurt fortified with marine fish oil, Journal of Dairy Science, 94 (12), 5760-5769 (2011)

Gracey, J.F, Collins, D.S. y Huey, R., Fat rancidity, en Meat Hygiene. pp 407, 10a edición Harcourt Brace and Company. Ltd., Londres, RU (1999)

González, R.E., Pérez, J.; Urbina, N.A., Efecto de la Microencapsulación sobre las Propiedades Reológicas y Fisicoquímicas del yogur blando. Información Tecnológica, 25(6), 45-56 (2014)

Kessler, H.G., The structure of fermented milk products as influenced by technology and composition, en Texture of Fermented Milk Products and Dairy Desserts, pp 93-105 IDF Special Issue 9802, Bruselas, Bélgica (1998)

Lebos, A.P., Beganovic, J., Kos. B., Buneta, A., Beluhan, S. y Suskovic, J., Influence of microencapsulation and transglutaminase on viability of probiotic strain Lactobacillus helveticus M92 and consistency of set yogurt, International Journal of Diary Technology, 64(2), 254-261 (2010)

McLennan, P.L. y Abeywardena, M.Y, Membrane basis for fish oil effects on the heart: linking natural hibernators to prevention of human sudden cardiac death, Journal of Membrane Biology, 206, 85-102 (2005)

Obatolu, V.A., Farinde, E.O., Adesetan, T.O. y Oladapo, M.O., Chemical and microbial properties of yogurt processed from cow's milk and soymilk, Journal of Food Processing and Preservation, 33, 245-254 (2009)

Özer, B.H. y Kirmaci, H.A., Functional milks and dairy beverages. International Journal of Dairy Technology, 63, 1$15(2010)$

Paseephol, T., Small, D.M. y Sherkat, F., Rheology and texture of set yogurt as affected by inulin addition, Journal of Texture Studies, 39, 617-634 (2008) 
Pirkul, T., Temiz, A. y Kemal, Y., Fortification of yoghurt with calcium salts and its effects on starter microorganism and yoghurt quality, International Dairy Journal, 11, 547-551 (1998)

Popov-Raljic, J.V., Lakié, N.S., Lalicic-Petronijevic, J.G., Baraé, M.B. y Sikimic, V.M., Color changes of UHT milk during storage, Sensors, 8, 5961- 5974 (2008)

Purwandari, U., Shah, N.P. y Vasiljevic, T., Effects of exopoly-saccharide-producing strains of Streptococcus thermophilus on technological and rheological properties of set-type yoghurt, International Dairy Journal, 17, 13441352 (2007)

Ramirez-Santiago, C., Ramos-Solis, L., Lobato-Calleros, C., Peña-Valdivia, C. y Vernon-Carter, E.J., Enrichment of stirred yogurt with soluble dietary fiber from Pachyrhizus erosus L. Urban: Effect on syneresis, microstructure and rheological properties. Journal of Food Engineering, 101, 229-235 (2010)

Ramírez-Sucre, M.O. y Vélez-Ruíz, J.F, The physicochemical and rheological properties of a milk drink flavoured with cajeta, a Mexican caramel jam, International J. Dairy Technology, 64(2), 294-304 (2011)

Ramírez-Sucre, M.O. y Vélez-Ruiz, J.F., Physicochemical; rheological and stability characterization of a caramel flavored yogurt. LWT - Food Science and Technology, Abril (1), 233-241 (2013)

Riediger, N.D., Othman, R.A., Suh, M. y Moghadasian, M.H., A systemic review of the roles of $n-3$ fatty acids in health and disease, Journal of the American Dietetic Association, 109, 668-679 (2009)

Saénz, C., Tapia, S., Chávez, J. y Robert, P., Microencapsulation by spray drying of bioactive compounds from cactus pear (Opuntia ficus-indica). Food Chemistry, 114, 61-622 (2009)

Sathivel, S. y Kramer, J.E., Microencapsulation, nanoencapsulation, edible film, and coating applications in seafood processing, Capítulo 33 en Handbook of Seafood Quality, Safety and Health Effects por Alasalvar, C., Miyashita, K., Shaidi F. y Wanasundara U., Blackwell Publishing Ltd., Oxford, RU (2010)

Sodini, I., Remeuf, F. y Haddad, S., The relative effect of milk base, starter and process on effects of milk fortification and heating on microstructure and physical properties of stirred yoghurts, International Dairy Journal, 13, 773-782 (2004)

Tamime, A.Y. y Robinson, R.K., Yogurt Science and Technology, 3a edición, pp 791, Woodhead Publishing Ltd. And CRC Press LLC, NY, EU (1999)

Tamjidi, F., Nasirpour, A. y Shahedi, M., Physicochemical and sensory properties of yogurt enriched with microencapsulated fish oil. doi: 10.1177/1082013211428212. Food Sci. \& Technol. Int. 18(4), 381-90 (2012)

Tang, Q., Munro, P.A. y McCarthy, O.J., Rheology of whey protein concentrate solutions as a function of concentration, temperature, $\mathrm{pH}$ and salt concentration. Journal of Dairy Research, 60, 349-361 (1993)

Tridjoko, W., Bouillannie, C., Landon, M. y Desmazeaud, M., Bacterial growth and volatile compounds in yoghurttype products from soymilk containing Bifidobacterium ssp, J. Food Science, 55, 153-155(1992)

US FDA-DHHS. 2011. 21CFR131.203: Low fat yogur. Acceso; Nov. (2014), http://www.accesdata.fda.gov/ scripts/cdrh/cfdcos/cfcfr/CFRSearch.cfm?, fr=131.203 (2011)

Valdés Martínez, S.E., Hidratos de carbono, en Química de los Alimentos por Badui Dergal, S. p 97-106 Pearson Educación de México, DF, México (2006)

Vélez-Ruiz, J.F., Rheological properties of yogurt, en Progress in Food Engineering Research and Development. pp 223-242 Ed. Nova Science Publishers, Inc. NY, EU (2008)

Vélez-Ruiz J.F. y Rivas, A.H., Revisión: Propiedades y características del yogur. Revista Internacional Información Tecnológica. 12 (6), 35-42 (2001)

Vélez-Ruiz, J.F, Hernández-Carranza, P. y Sosa-Morales, M.E., Physicochemical and flow properties of low fat yogurt fortified with calcium and fiber. Journal of Food Processing and Preservation, 37, 210-221 (2012)

Weitz, D., Weintraub, H., Fisher, E. y Schwartzbard, A.Z., Fish oil for the treatment of cardiovascular disease. Cardiological in Review, 18, 258-263 (2010)

Wendel, M. y Heller, A.R., Anticancer action of omega-3 fatty acids current state and future perspectives. Anticancer Agents and Medicinal Chemistry, 9, 457-470 (2009) 